\title{
The Therapeutic Outcomes of Mechanical Ventilation in Hematological Malignancy Patients with Respiratory Failure
}

\author{
Yusuke Fujiwara ${ }^{1}$, Hiroki Yamaguchi ${ }^{1}$, Katsuya Kobayashi ${ }^{2}$, Atsushi Marumo ${ }^{1}$, \\ Ikuko Omori ${ }^{1}$, Satoshi Yamanaka ${ }^{1}$, Shunsuke Yui ${ }^{1}$, Keiko Fukunaga ${ }^{1}$, Takeshi Ryotokuji ${ }^{1}$, \\ Tsuneaki Hirakawa ${ }^{1}$, Masahiro Okabe ${ }^{1}$, Satoshi Wakita ${ }^{1}$, Hayato Tamai ${ }^{1}$, Muneo Okamoto ${ }^{1}$, \\ Kazutaka Nakayama ${ }^{1}$, Shinhiro Takeda ${ }^{2}$ and Koiti Inokuchi ${ }^{1}$
}

\begin{abstract}
Objective In hematological malignancy patients, the complication of acute respiratory failure often reaches a degree of severity that necessitates mechanical ventilation. The objective of the present study was to investigate the therapeutic outcomes of mechanical ventilation in hematological malignancy patients with respiratory failure and to analyze the factors that are associated with successful treatment in order to identify the issues that should be addressed in the future.

Methods The present study was a retrospective analysis of 71 hematological malignancy patients with noncardiogenic acute respiratory failure who were treated with mechanical ventilation at Nippon Medical School Hospital between 2003 and 2014.

Results Twenty-six patients $(36.6 \%)$ were treated with mechanical ventilation in an intensive care unit (ICU). Non-invasive positive pressure ventilation (NPPV) was applied in 29 cases $(40.8 \%)$. The rate of successful mechanical ventilation treatment with NPPV alone was $13.8 \%$. The rate of endotracheal extubation was $17.7 \%$. A univariate analysis revealed that the following factors were associated with the successful extubation of patients who received invasive mechanical ventilation: respiratory management in an ICU ( $p=$ $0.012)$; remission of the hematological disease $(p=0.011)$; female gender $(p=0.048)$; low levels of accompanying non-respiratory organ failure $(\mathrm{p}=0.041)$; and the non-use of extracorporeal circulation $(\mathrm{p}=0.005)$. A subsequent multivariate analysis revealed that respiratory management in an ICU was the only variable associated with successful extubation $(\mathrm{p}=0.030)$.

Conclusion The outcomes of hematological malignancy patients who receive mechanical ventilation treatment for respiratory failure are very poor. Respiratory management in an ICU environment may be useful in improving the therapeutic outcomes of such patients.
\end{abstract}

Key words: hematological malignancy, respiratory failure, mechanical ventilation, intensive care unit, sterile ward

(Intern Med 55: 1537-1545, 2016)

(DOI: 10.2169/internalmedicine.55.5822)

\section{Introduction}

Acute respiratory failure, which is the most frequent or- gan failure complication in hematological malignancy patients, is associated with a poor prognosis (1-3). In hematological malignancy patients, the complication of acute respiratory failure frequently requires mechanical ventilation,

${ }^{1}$ Department of Hematology, Nippon Medical School, Japan and ${ }^{2}$ Department of Intensive Care Medicine, Nippon Medical School Hospital, Japan

Received for publication May 25, 2015; Accepted for publication September 14, 2015

Correspondence to Dr. Hiroki Yamaguchi, y-hiroki@fd6.so-net.ne.jp 
which is associated with a hospital mortality rate of 70$75 \%$ (4).

The causes of acute respiratory failure in hematological malignancy patients are varied, but are broadly divided into infection-related and non-infection-related etiologies. The causes of infection-related respiratory failure include bacterial infection, invasive pulmonary aspergillosis and other invasive fungal infections, and viral infections. The causes of non-infection-related respiratory failure include acute heart failure, hemorrhage, drug-related toxicity, radiation-induced lung dysfunction, malignant cell infiltration, and graft versus host disease. In hematological malignancy patients with acute respiratory failure, a definitive diagnosis often requires invasive testing, as it can be difficult to determine the cause of the complication via diagnostic imaging alone (5). However, the depleted white blood cell and platelet counts of such patients (due to the original disease and its treatment) often prevents invasive testing and the identification of the cause. As neutrophils are also depleted, the therapeutic response in infection-related cases is often poor and the treatment protracted and difficult $(6,7)$.

In many hematological malignancy patients, acute respiratory failure reaches a degree of severity that necessitates mechanical ventilation. However, physicians often have great misgivings about deciding to introduce mechanical ventilation. This is because the use of endotracheal intubation and mechanical ventilation (invasive mechanical ventilation: IMV) in hematological malignancy patients carries a high risk of ventilator-associated pneumonia (VAP) and because it was not actively recommended in the past (8). Recently, non-invasive positive pressure ventilation (NPPV), which can also be used in sterile wards, has come into widespread use. It appears that (in comparison to IMV) the use of NPPV may reduce the risk of VAP in immunosuppressed patients who are treated for respiratory failure and shorten the duration of mechanical ventilation (9), thus avoiding progression to IMV and reducing the rate of intensive care unit (ICU) admission (10). Thus there are reports which assert that acute respiratory failure in hematological malignancy patients should initially be treated with NPPV (11). However, there are conflicting reports which state that NPPV is not superior to IMV as an initial treatment for acute respiratory failure in hematological malignancy patients $(12,13)$. In hematological malignancy patients, acute respiratory failure is often accompanied by other forms of organ failure, thus NPPV alone is often insufficient and IMV treatment will eventually become necessary (14). Furthermore, it has been reported that in many of the cases in which the initial treatment with NPPV fails, it is difficult to wean the patient from mechanical ventilation and that the prognosis of such patients is poor; thus the decision to use NPPV as the initial treatment should not be taken lightly (15).

In hematological malignancy patients, the cause of respiratory failure is difficult to diagnose due to the presence of immunodeficiency and hemorrhagic tendency, and treatment resistance frequently develops. As a result, the clinical profile of Japanese hematological malignancy patients with respiratory failure has not been clearly established. There are also many areas of uncertainty in relation to the treatment of these patients, including the administration of IMV and NPPV. The objective of the present study was to investigate the therapeutic outcomes of mechanical ventilation in Japanese hematological malignancy patients with respiratory failure and to analyze the factors associated with successful treatment in order to identify the issues that should be addressed going forward.

\section{Materials and Methods}

The present study was a retrospective analysis of the cases of 76 hematological malignancy patients who received mechanical ventilation for acute respiratory failure at Nippon Medical School Hospital between 2003 and 2014. Cases in which there was no prospect of curative treatment due to treatment resistance in the underlying disease and cases in which the patient or the patient's family refused ICU treatment, mechanical ventilation treatment, or extracorporeal circulation were excluded from the study. Respiratory failure was defined as an arterial oxygen partial pressure of less than $60 \mathrm{mmHg}$. Eligible cases were those of rapid onset respiratory failure where, regardless of the cause, mechanical ventilation was applied due to continued hypoxemia despite adequate oxygen administration.

A total of 76 patients received mechanical ventilation for respiratory failure. Five patients, in whom a cardiogenic etiology was definitely diagnosed, were excluded from the study. The remaining 71 patients were included in the present study. The term, "non-cardiogenic respiratory failure," was defined according to the definition of acute lung injury (ALI)/acute respiratory distress syndrome (ARDS) as issued by the American-European Consensus Conference (AECC: a joint consultative body formed by the American Thoracic Society and the European Society of Intensive Care Medicine) as a condition arising from an underlying disease in which cardiogenic pulmonary edema can be excluded and characterized by acute hypoxemia and bilateral pulmonary infiltrates that are visible in X-ray imaging. The term, "mechanical ventilation," included both IMV and NPPV. Cases were only eligible when mechanical ventilation was sustained for at least 24 hours.

To assess organ failure in the eligible cases in the present study, the presence of organ failure was evaluated based on the patients' laboratory data. The laboratory data used were the data measured within 24 hours of the start of mechanical ventilation. The ongoing measurement of the hematological data continued after the start of mechanical ventilation. Hepatic failure was recognized when hepatic dysfunction was indicated by the following values: aspartate aminotransferase $($ AST) $>20 \mathrm{IU} / \mathrm{L}$, alanine aminotransferase $($ ALT) $>20 \mathrm{IU} / \mathrm{L}$, and total bilirubin $>2.0 \mathrm{mg} / \mathrm{dL}$. Renal failure was recognized in cases of renal dysfunction when the serum creatinine 
level was $>2.0 \mathrm{mg} / \mathrm{dL}$. Heart failure was recognized when there were clear symptoms of heart failure and a brain natriuretic peptide (BNP) level of $>100 \mathrm{pg} / \mathrm{mL}$.

The primary endpoint was to identify the factors that were associated with successful endotracheal extubation in non-cardiogenic respiratory failure patients. The patients' clinical data were subjected to statistical analyses using Chisquare, Fisher, and $t$ tests. The cumulative incidence of endotracheal extubation and mechanical ventilator weaning was analyzed using the Kaplan-Meier log-rank test. p values of $<0.05$ were considered to indicate statistical significance. A multivariate analysis, using Cox's proportional hazards model, was conducted to identify the factors that were associated with successful endotracheal extubation. A stepwise backward procedure selection model was used to extract independent events. Events with a significance level of $\mathrm{p} \leq 0.15$ were included in the analysis. The IBM SPSS Statistics software program (version 21.0.0.0, IBM, New York, USA) was used for all of the statistical analyses.

\section{Results}

\section{Mechanical ventilation for respiratory failure in he- matological malignancy patients}

Among the 71 hematological disease patients with noncardiogenic respiratory failure, $45(63.4 \%)$ patients were treated with mechanical ventilation in a sterile ward, while $26(36.6 \%)$ received mechanical ventilation in an ICU. Among the patients who received mechanical ventilation, 62 $(87.3 \%)$ received IMV, while $29(40.8 \%)$ received NPPV, and $20(28.2 \%)$ received both NPPV and endotracheal intubation. The average durations of mechanical ventilation, intubation and NPPV were 12.5 days, 12.2 days, and 5.2 days, respectively. The rate of endotracheal extubation was $17.7 \%(\mathrm{n}=11)$, and the rate of weaning from mechanical ventilation was $25.4 \%(n=18)$. Fifty-three $(74.6 \%)$ patients died without being weaned from mechanical ventilation.

\section{The identification of the factors associated with suc- cessful endotracheal extubation in non-cardiogenic respiratory failure patients}

From the total population of 71 patients who received mechanical ventilation treatment, the cases of 62 patients who underwent intubation were analyzed in order to identify the factors that were significantly associated with successful extubation (Table 1). Mechanical ventilation in an ICU ( $\mathrm{p}=$ $0.012)$ and remission of the underlying disease $(p=0.011)$ were identified as significant factors.

\section{The backgrounds of the patients in the sterile ward and ICU treatment groups}

The background of hematological malignancy in the patients with non-cardiogenic respiratory failure was compared in the sterile ward group and the ICU group (Table 2). The ICU treatment group had a significantly higher number of male patients $(\mathrm{p}=0.034)$, patients who received both intubation and NPPV (sterile ward group: $5 / 45$ cases, $11.1 \%$; ICU group: $15 / 26$ cases, $57.7 \%$; $<<0.001$ ), and patients who were treated between 2009 and 2014 (sterile ward group: 16/45 cases, 35.6\%; ICU group: $21 / 26$ cases, $80.8 \%$; $\mathrm{p}=0.001$ ). No significant intergroup differences were found in the factors that were considered likely to affect the therapeutic course (i.e. the number of chemotherapy sessions before the introduction of mechanical ventilation and the neutrophil and platelet counts).

The most frequent cause of non-cardiogenic respiratory failure was bacterial pneumonia [25/71 cases (35.2\%)] (Table 3). There were no differences in the causes of noncardiogenic respiratory failure in the sterile ward and ICU groups.

\section{The usefulness of NPPV against non-cardiogenic respiratory failure}

Among the 29 patients who received NPPV, 9 (31.0\%) patients received mechanical ventilation through NPPV alone. Four of these patients (13.8\%) were successfully treated with NPPV alone. NPPV was used more frequently for the treatment of non-cardiogenic respiratory failure in the ICU group (sterile ward group: 10/45 cases, 22.2\%; ICU group: $19 / 26$ cases, $73.1 \%$; $p<0.001$ ). There was no significant difference in the treatment outcomes of the patients who were treated with NPPV only (sterile ward group: 1/5 cases, 20.0\%; ICU group: $3 / 4$ cases, 75.0\%; p=0.206). In both groups, all cases of unsuccessful treatment progressed to mechanical ventilation based on endotracheal intubation.

\section{Assessment of non-respiratory organ failure}

Acute respiratory failure in hematological malignancy patients is frequently accompanied by other forms of organ failure. With non-cardiogenic respiratory failure, the sterile ward group had dysfunction in an average of 0.622 organs, while the ICU group had dysfunction in an average of 1.000. There was no distinctive profile in the organs that were affected. The number of dysfunctional organs tended to be higher in the ICU group $(\mathrm{p}=0.055)$ (Table 2). The number of cases that required extracorporeal circulation [such as patients who received continuous renal replacement therapy (CRRT)] was significantly higher in the ICU group $(11 / 26$ cases, $42.3 \%)$ than in the sterile ward group $(3 / 45$ cases, 6.7\%) $(\mathrm{p}<0.001)$ (Table 2). All of the patients in the ICU group who underwent extracorporeal circulation, received continuous renal replacement therapy and none of the patients in the ICU group received hemodialysis (Table 4). There were no intergroup differences in the hematological data from the time that extracorporeal circulation was introduced (Table 4).

\section{The rates of endotracheal extubation and weaning from mechanical ventilation}

The rate of endotracheal extubation was significantly higher in the ICU group than in the sterile ward group (ster- 
Table 1. Investigation of Factors in Successful Endotracheal Extubation among Hematological Malignancy Patients with Complicating Acute Respiratory Failure.

\begin{tabular}{|c|c|c|c|}
\hline & Success $(n=11)$ & Failure $(n=51)$ & $\mathrm{p}$ \\
\hline Sterile ward : ICU & $3: 8$ & $37: 14$ & 0.012 \\
\hline Age (range) & $55(24-82)$ & $61(21-85)$ & 0.133 \\
\hline Sex male: female & $7: 4$ & $38: 13$ & 0.475 \\
\hline \multicolumn{4}{|l|}{ Hematological disease } \\
\hline Acute myeloid leukemia & 4 & 14 & 0.716 \\
\hline Myelodysplastic syndrome & 3 & 9 & 0.432 \\
\hline Acute lymphoid leukemia & 0 & 3 & 1.000 \\
\hline Malignant lymphoma & 2 & 17 & 0.478 \\
\hline Other & 2 & 7 & 0.655 \\
\hline Chronic lymphocytic leukemia & 1 & 0 & - \\
\hline Multiple myeloma & 1 & 0 & - \\
\hline Aplastic anemia & 0 & 1 & - \\
\hline Hairy cell leukemia & 0 & 1 & - \\
\hline Idiopathic thrombocytopenic purpura & 0 & 1 & - \\
\hline Myelofibrosis & 0 & 2 & - \\
\hline Hemophagocytic syndrome & 0 & 1 & - \\
\hline Myeloproliferative neoplasma & 0 & 1 & - \\
\hline $\begin{array}{l}\text { Number of chemotherapy sessions prior to introduction } \\
\text { of mechanical ventilation }\end{array}$ & $3.143 \pm 1.053$ & $3.563 \pm 0.796$ & 0.792 \\
\hline Remission vs. non-remission & $5: 6$ & $5: 46$ & 0.011 \\
\hline HSCT & 5 & 18 & 0.732 \\
\hline \multicolumn{4}{|l|}{ Main cause of acute respiratory failure } \\
\hline Infiltration & 1 & 6 & 1.000 \\
\hline Bacterial & 3 & 22 & 0.501 \\
\hline Fungal & 1 & 5 & 1.000 \\
\hline Viral & 2 & 2 & 0.141 \\
\hline Drug-related & 1 & 4 & 1.000 \\
\hline Other & 3 & 12 & 1.000 \\
\hline Hemorrhage & 1 & 0 & - \\
\hline Pleural effusion & 1 & 0 & - \\
\hline Obstructive & 0 & 1 & - \\
\hline Tumor lysis syndrome & 0 & 2 & - \\
\hline Interstitial pneumonia & 0 & 1 & - \\
\hline Lung GVHD & 0 & 1 & - \\
\hline Pneumocystis jirovecii & 0 & 6 & - \\
\hline Unknown & 1 & 1 & - \\
\hline Neutrophil $(/ \mu \mathrm{L})$ & $2,864 \pm 691.0$ & $9,803 \pm 2,504$ & 0.207 \\
\hline Platelet $\left(\times 10^{3} / \mu \mathrm{L}\right)$ & $6.527 \pm 1.522$ & $5.439 \pm 1.134$ & 0.671 \\
\hline Number of organ failures & $0.700 \pm 0.260$ & $0.735 \pm 0.112$ & 0.900 \\
\hline CRRT & 1 & 11 & 0.675 \\
\hline Historical treatment period (2003-2008 vs. $2009-2014)$ & $4: 7$ & $28: 23$ & 0.329 \\
\hline
\end{tabular}

ile ward group: 3/40 cases, 7.5\%; ICU group: 8/22 cases, $36.3 \%$; $=0.012$ ) (Figure A). The rate of weaning from mechanical ventilation, including NPPV, was also significantly higher in the ICU group than in the sterile ward group (sterile ward group: $7 / 45$ cases, $15.6 \%$; ICU group: 11/26 cases, $42.3 \%$; $\mathrm{p}=0.022$ ) (Figure B). The 28-day cumulative rate of endotracheal extubation (sterile ward group: 3/40 cases, 7.5\%; ICU group: $6 / 22$ cases, $36.2 \%$; $=0.239$ ) and the cumulative mechanical ventilator weaning rate (sterile ward group: 6/45 cases, 23.9\% ; ICU group: 9/26 cases, $43.1 \%$; $\mathrm{p}=0.285$ ) were also higher in the ICU group than in the sterile ward group, but not to a statistically significant extent (Figure C, D). These findings indicate that the treatment of the acute respiratory failure in an ICU produced better treatment outcomes in hematological malignancy patients with non-cardiogenic respiratory failure.

\section{The efficacy of mechanical ventilation treatment in an ICU}

In the ICU group, the factors that were associated with successful endotracheal extubation were identified by comparing the patients who were successfully extubated with those who were not (Table 5). The following significant factors were identified in the patients in whom endotracheal extubation was successful: female gender $(\mathrm{p}=0.048)$; low levels of accompanying non-respiratory organ failure $(\mathrm{p}=0.041)$; and the non-use of extracorporeal circulation ( $\mathrm{p}=0.005)$. Regarding the time from when the need for mechanical ventilation arose (within 24 hours or after $\geq 24$ hours) until ICU admission, no difference was found in the rate of successful endotracheal extubation in the patients who were admitted to the ICU within 24 hours and the patients who were admitted after $\geq 24$ hours (within 24 hours: $2 / 7$ cases, $28.6 \%$; 
Table 2. Patient Background in Non-cardiogenic Respiratory Failure as a Complication of Hematological Malignancy Patients.

\begin{tabular}{|c|c|c|c|}
\hline & Sterile ward $(n=45)$ & $\operatorname{ICU}(n=26)$ & $\mathbf{p}$ \\
\hline Age (range) & $62(30-85)$ & $56.5(20-81)$ & 0.076 \\
\hline Sex (male: female) & $31: 14$ & $21: 5$ & 0.034 \\
\hline \multicolumn{4}{|l|}{ Hematological disease } \\
\hline Acute myeloid leukemia & 11 & 11 & 0.182 \\
\hline \multicolumn{4}{|l|}{ FAB } \\
\hline M0 & 0 & 3 & - \\
\hline M1 & 1 & 1 & - \\
\hline M2 & 3 & 4 & - \\
\hline M3 & 2 & 2 & - \\
\hline M5 & 3 & 1 & - \\
\hline Myeloid/Nkcell precursor acute leukemia & 1 & 0 & - \\
\hline Unknown & 1 & 0 & - \\
\hline Myelodysplastic syndrome & 8 & 5 & 1.000 \\
\hline MDS overt leukemia & 4 & 3 & - \\
\hline RAEB2 & 1 & 1 & - \\
\hline RAEB1 & 0 & 1 & - \\
\hline RCMD & 1 & 0 & - \\
\hline RA & 2 & 0 & - \\
\hline Acute lymphoid leukemia & 3 & 2 & 1.000 \\
\hline Malignant lymphoma & 14 & 4 & 0.168 \\
\hline Diffuse large B cell lymphoma & 5 & 1 & - \\
\hline Burkitt lymphoma & 0 & 1 & - \\
\hline Peripheral Tcell lymphoma & 3 & 0 & - \\
\hline Angioimmunoblastic T cell lymphoma & 0 & 1 & - \\
\hline Mantle cell lymphoma & 1 & 0 & - \\
\hline Follicular small B cell lymphoma & 1 & 0 & - \\
\hline Primary effusion lymphoma & 1 & 0 & - \\
\hline Splenic marginal zone lymphoma & 0 & 1 & - \\
\hline Enteropathy associated $\mathrm{T}$ cell lymphoma & 1 & 0 & - \\
\hline B cell lymphoma (unknown) & 2 & 0 & - \\
\hline Other & 9 & 4 & 0.756 \\
\hline Multiple myeloma & 1 & 1 & - \\
\hline Chronic lymphocytic leukemia & 1 & 0 & - \\
\hline Chronic myeloid leukemia & 1 & 0 & - \\
\hline Adult T cell leukemia (acute type) & 1 & 0 & - \\
\hline Myeloproliferative disease (unknown) & 1 & 0 & - \\
\hline Aplastic anemia & 1 & 0 & - \\
\hline Secondary myelofibrosis (unknown) & 1 & 1 & - \\
\hline Hairy cell leukemia & 1 & 0 & - \\
\hline Idiopathic thrombocytopenic purpura & 1 & 0 & - \\
\hline Castleman's disease & 0 & 1 & - \\
\hline Hemophagocytic syndrome & 0 & 1 & - \\
\hline $\begin{array}{l}\text { Number of chemotherapy sessions prior to introduction } \\
\text { of mechanical ventilation }\end{array}$ & $3.154 \pm 0.731$ & $3.778 \pm 0.832$ & 0.613 \\
\hline Remission vs. non-remission & $5: 40$ & $5: 21$ & 0.481 \\
\hline HSCT & 16 & 9 & 1.000 \\
\hline Neutrophil $(/ \mu \mathrm{L})$ & $10,040 \pm 2,879$ & $4,912 \pm 1,014$ & 0.099 \\
\hline Platelet $\left(\times 10^{3} / \mu \mathrm{L}\right)$ & $6.402 \pm 1.346$ & $5.608 \pm 1.493$ & 0.694 \\
\hline Number of organ failures & $0.622 \pm 0.107$ & $1.000 \pm 0.175$ & 0.055 \\
\hline CRRT & 3 & 11 & $<0.001$ \\
\hline Historical treatment period (2003-2008 vs. 2009-2014) & $29: 16$ & $5: 21$ & 0.001 \\
\hline IMV alone & 35 & 7 & $<0.001$ \\
\hline NPPV alone & 5 & 4 & 0.716 \\
\hline Use of both IMV and NPPV & 5 & 15 & $<0.001$ \\
\hline
\end{tabular}

after $\geq 24$ hours: $9 / 19$ cases, $47.4 \%$; $\mathrm{p}=0.658$ ).

In order to establish the importance of these factors, including the differences in the background characteristics of the patients, we conducted multivariate a analysis to assess their effect on successful endotracheal extubation. A multivariate analysis using Cox's proportional hazards models revealed that treatment in an ICU $(\mathrm{p}=0.030)$ was a significant independent factor in successful endotracheal extubation
(Table 6)

\section{Discussion}

The present study analyzed the therapeutic outcomes of mechanical ventilation in Japanese hematological malignancy patients with respiratory failure and the associated prognostic factors. 
Table 3. Cause of Non-cardiogenic Respiratory Failure in Hematological Malignancy Patients.

\begin{tabular}{lcrr}
\hline & Sterile ward $(\mathrm{n}=45) \mathrm{ICU}(\mathrm{n}=26)$ & $\mathrm{p}$ \\
\hline Infiltration & 5 & 3 & 1.000 \\
Bacterial & 17 & 8 & 0.613 \\
Fungal & 7 & 5 & 0.748 \\
Viral & 1 & 3 & 0.136 \\
Drug-related & 3 & 4 & 0.410 \\
Other & 12 & 3 & 0.227 \\
Hemorrhage & 0 & 1 & - \\
Pleural effusion & 1 & 0 & - \\
Obstructive & 1 & 0 & - \\
Tumor lysis syndrome & 2 & 0 & - \\
Interstitial pneumonia & 1 & 0 & - \\
Lung GVHD & 1 & 0 & - \\
Pneumocystis jirovecii & 5 & 1 & - \\
CO ${ }_{2}$ narcosis & 1 & 0 & - \\
Unknown & 0 & 1 & - \\
\hline GVHD: graft versus host disease, $\mathrm{CO}_{2}:$ carbon dioxide &
\end{tabular}

A

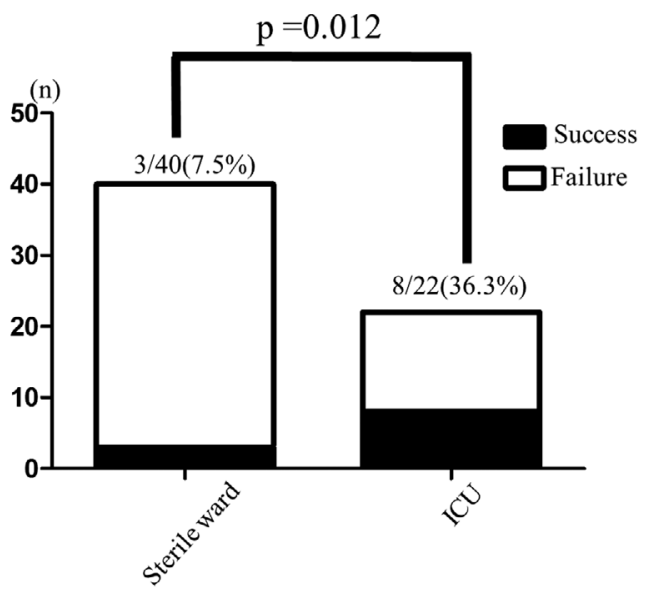

C

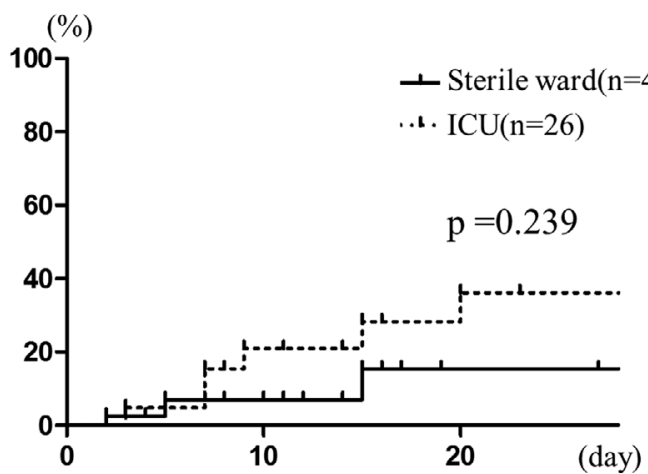

Table 4. Data on Introduction of Extracorporeal Circulation in Hematological Malignancy Patients with Multiple Organ Failure Receiving Mechanical Ventilation.

\begin{tabular}{|c|c|c|c|}
\hline & sterile ward $(n=3)$ & $\operatorname{ICU}(\mathrm{n}=11)$ & $\mathrm{p}$ \\
\hline Age (range) & $62(56-67)$ & $56.5(20-81)$ & 0.444 \\
\hline Sex (male:female) & $2: 1$ & $11: 0$ & 0.214 \\
\hline CRRT:HD & $1: 2$ & $11: 0$ & 0.033 \\
\hline \multicolumn{4}{|c|}{ Blood biochemistry test } \\
\hline BUN (mg/dL) & $58.90 \pm 21.78$ & $86.77 \pm 14.77$ & 0.383 \\
\hline Cre $(\mathrm{mg} / \mathrm{dL})$ & $4.613 \pm 0.835$ & $4.05 \pm 1.038$ & 0.791 \\
\hline AST (U/L) & $896.0 \pm 869.0$ & $423.2 \pm 225.1$ & 0.444 \\
\hline $\operatorname{ALT}(\mathrm{U} / \mathrm{L})$ & $453.3 \pm 436.9$ & $432.7 \pm 325.0$ & 0.976 \\
\hline T-Bil (mg/dL) & $1.333 \pm 0.267$ & $4.80 \pm 2.213$ & 0.443 \\
\hline $\begin{array}{l}\text { CRRT: continuc } \\
\text { hemodialysis, BUI } \\
\text { aspartate aminotra } \\
\text { bil: total bilirubin }\end{array}$ & renal replaceme & $\begin{array}{l}\text { nt therapy, } \\
\text { Cre: creatine, } \\
\text { aminotransfer }\end{array}$ & $\begin{array}{l}\text { HD: } \\
\text { AST: } \\
\text { se, T- }\end{array}$ \\
\hline
\end{tabular}

B

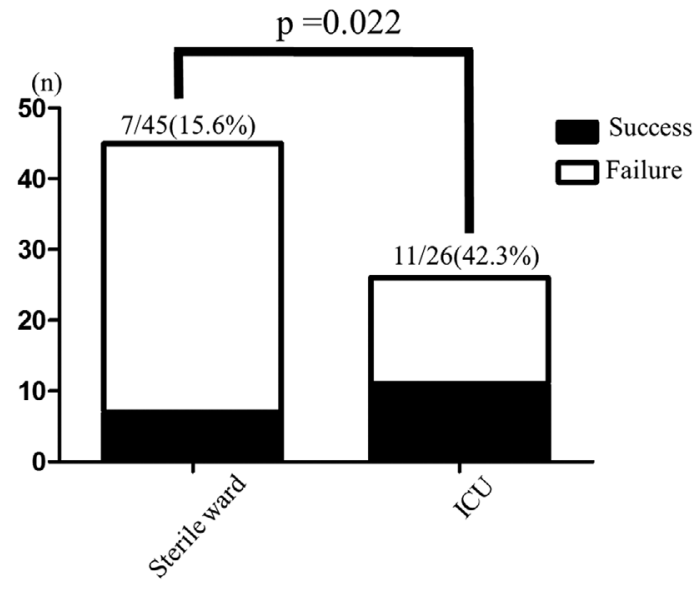

$\mathrm{D}$

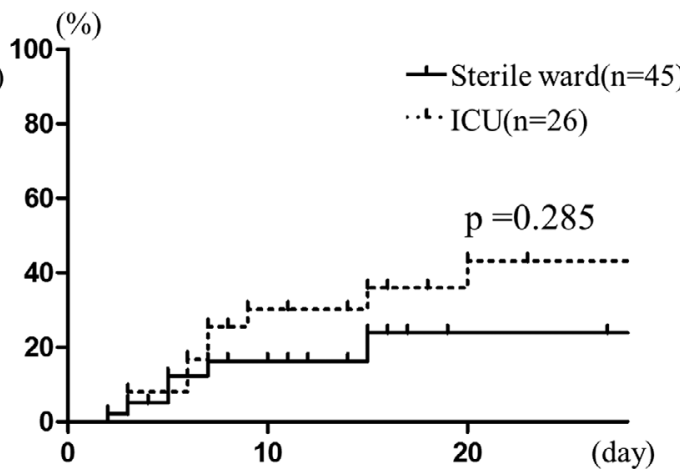

Figure. The treatment outcome of mechanical ventilation in hematological malignancy patients with acute respiratory failure. A: A comparison of the endotracheal extubation rates in the sterile ward and ICU groups. B: A comparison of the mechanical ventilation weaning rates in the sterile ward and ICU groups. C: The 28-day cumulative endotracheal extubation rates in the sterile ward and ICU groups. D: The 28-day cumulative rate of weaning from mechanical ventilation in the sterile ward and ICU groups. 
Table 5. Identification of Factors in Successful Endotracheal Extubation during ICU Treatment of Hematological Malignancy Patients with Complicating Acute Respiratory Failure.

\begin{tabular}{|c|c|c|c|}
\hline & Success $(n=11)$ & Failure $(n=15)$ & $\mathrm{p}$ \\
\hline Age, (median) & $55(24-81)$ & $61(21-81)$ & 0.867 \\
\hline Sex (male:female) & $7: 4$ & $14: 1$ & 0.048 \\
\hline \multicolumn{4}{|l|}{ Hematological disease } \\
\hline Acute myeloid leukemia & 5 & 6 & 1.000 \\
\hline Myelodysplastic syndrome & 3 & 2 & 0.620 \\
\hline Acute lymphoid leukemia & 0 & 2 & 0.492 \\
\hline Malignant lymphoma & 1 & 3 & 0.614 \\
\hline Other & 2 & 2 & 1.000 \\
\hline Multiple myeloma & 1 & 0 & - \\
\hline Myelofibrosis & 0 & 1 & - \\
\hline Hemophagocytic syndrome & 0 & 1 & - \\
\hline Castleman's disease & 1 & 0 & - \\
\hline $\begin{array}{l}\text { Number of chemotherapy sessions prior to introduction } \\
\text { of mechanical ventilation }\end{array}$ & $3.182 \pm 1.182$ & $3.133 \pm 0.9605$ & 0.975 \\
\hline Remission vs. non-remission & $4: 1$ & $1: 14$ & 0.128 \\
\hline HSCT & 4 & 5 & 1.000 \\
\hline \multicolumn{4}{|l|}{ Main cause of acute respiratory failure } \\
\hline Infiltration & 0 & 3 & 0.239 \\
\hline Bacterial & 5 & 3 & 1.000 \\
\hline Fungal & 0 & 5 & 0.053 \\
\hline Viral & 2 & 1 & 0.556 \\
\hline Drug-related & 2 & 2 & 1.000 \\
\hline Other & 2 & 1 & 1.000 \\
\hline Hemorrhage & 1 & 0 & - \\
\hline Pneumocystis jirovecii & 0 & 1 & - \\
\hline Unknown & 1 & 0 & - \\
\hline Neutrophil $(/ \mu \mathrm{L})$ & $3,510 \pm 1,442$ & $3,701 \pm 1,195$ & 0.920 \\
\hline Platelet $\left(\times 10^{3} / \mu \mathrm{L}\right)$ & $8.182 \pm 3.388$ & $3.720 \pm 0.535$ & 0.223 \\
\hline Number of organ failures & $0.909 \pm 0.163$ & $1.600 \pm 0.273$ & 0.041 \\
\hline CRRT & 1 & 10 & 0.005 \\
\hline Historical treatment period (2003-2008 vs. 2009-2014) & $3: 8$ & $2: 13$ & 0.620 \\
\hline
\end{tabular}

Table 6. Multivariate Analyses with Cox Proportional Hazard Models for Identification of Independent Factors in Successful Endotracheal Extubation.

\begin{tabular}{llllr}
\hline \multicolumn{1}{c}{ Factor } & HR & $\mathrm{p}$ & \multicolumn{2}{c}{$95.0 \%$ CI } \\
\hline Treatment in ICU & 3.991 & 0.030 & 1.136 & 14.02 \\
Female & 0.644 & 0.491 & 0.184 & 2.250 \\
Remission & 1.992 & 0.364 & 0.449 & 8.829 \\
Number of organ failures $\leq 1$ & 1.555 & 0.654 & 0.226 & 10.719 \\
Non-use of CRRT & 6.026 & 0.114 & 0.650 & 55.844 \\
Recent case (2009-2014) & 0.975 & 0.969 & 0.263 & 3.616 \\
Use of both IMV and NPPV & 0.410 & 0.203 & 0.104 & 1.617 \\
\hline HR: hazard ratio, CI: confidence interval, ICU: intensive care \\
unit, CRRT: continuous renal replacement therapy, IMV: \\
invasive mechanical ventilation, NPPV: non invasive positive \\
pressure
\end{tabular}

In hematological malignancy patients with acute respiratory failure, treatment with NPPV alone was rarely successful (sterile ward group 2.2\%, ICU group 11.5\%); however, concerns over the invasiveness of IMV may have caused a delay in progression to this therapy. In recent years, NPPV is more frequently utilized in general wards, including sterile wards, and there are reports that indicate that it is useful for treating non-cardiogenic respiratory failure in patients with hematological malignancy or immunodeficiency (11). Thus, the present study also investigated the usefulness of NPPV. NPPV was more frequently used to treat non- cardiogenic respiratory failure in the ICU group $(\mathrm{p}<0.001)$. It is hypothesized that the reason for this is that, in cases where accompanying VAP or difficult extubation is predicted, past reports (8) have led to the expectation that improvement is more likely to be achieved with NPPV than IMV; thus NPPV was more frequently applied in the ICU treatment group (10). However, the severity of acute respiratory failure as a complication of hematological malignancy makes it difficult to manage acute respiratory failure with mechanical ventilation via NPPV alone (16). Consequently, almost all of the cases progressed to IMV. (9)

A large number of issues are associated with the use of IMV for non-cardiogenic respiratory failure in hematological malignancy patients. A sterile ward is an isolated individual room. Because of this, continuous monitoring is not possible and there are limits to the management of the ventilator by the nursing staff. Questions arise as to whether clinicians are able to carry out effective respiratory management in an environment with insufficient monitoring, and whether they are able to adequately manage the artificial ventilator, which they use infrequently. In contrast, the ICU is an environment in which IMV can be applied by intensive care doctors accustomed to providing mechanical ventilation and who can monitor the patient continuously with the support of adequate nursing resources. Although NPPV also appears to be 
useful in the initial stage of acute respiratory failure, treatment with NPPV alone was unsuccessful in the majority of cases, and the failure of the initial NPPV treatment may be associated with a prognosis (14). The above results appear to indicate a need for stringent monitoring to ensure that there is no delay in the progression from NPPV to IMV, and that an ICU is a preferable treatment environment to a sterile ward (17).

Organ failure as a complication of hematological malignancy is a severe condition (1), and may require CRRT or other forms of extracorporeal circulation in addition to IMV. However, when hematological malignancy is accompanied by organ failure that is severe enough to require extracorporeal circulation, the weaning rate from extracorporeal circulation is low $(7.4 \%)$. In the present study, death occurred in all of the cases in which weaning failed. Extracorporeal circulation was thus associated with a very poor prognosis. This is thought to be due not only to the presence of severe organ failure itself, but also to other factors, including the increased risk of catheter-related infections due to long-term catheter use in patients who are susceptible to infection, the risk of taking an invasive approach in patients with hemorrhagic tendency, and the risk of multiple new complications arising from the use of extracorporeal circulation. In other words, weaning from IMV appears to be extremely difficult in hematological malignancy patients with non-cardiogenic respiratory failure and organ failure (other than respiratory failure) that is severe enough to require extracorporeal circulation.

In the present study, a retrospective analysis was undertaken to identify the factors that were associated with successful endotracheal extubation in hematological malignancy patients with acute respiratory failure. However, the present study was associated with some limitations. It was retrospective in nature, was performed at a single center with a small study population and the amount of data was insufficient. Furthermore, the possibility of bias needs to be considered with regard to the conclusion that the therapeutic outcomes of mechanical ventilation are better in ICUs. To reduce the bias in patient selection, patients were excluded from the analysis if their underlying disease offered no prospect of cure, or when the patient or the patient's family did not give permission for intensive care treatment or extracorporeal circulation. To account for intergroup bias, a multivariate analysis was carried out to cover the other factors that were associated with successful extubation (Table 1), and to cover the background factors (Table 2) that were associated with significant differences in the outcome of patients in the ICU and sterile ward groups. However, a number of major issues remained. Among these were the differences in the criteria that the different attending physicians used to refer patients to an ICU, the vastly greater ratio of nursing staff to patients in ICUs in comparison to sterile wards, the fact that the study did not account for the marked advances in drugs, medical equipment, and other resources over the last ten years (although it did include the historical period of treatment as a factor in the multivariate analysis), and the fact that blood gas, ventilator settings, and other factors were not analyzed. Going forward, there is a need for a prospective multi-center joint study of ICUs to verify the findings of the present study and to identify other factors that are associated with successful endotracheal extubation.

\section{The authors state that they have no Conflict of Interest (COI).}

Yusuke Fujiwara and Hiroki Yamaguchi contributed equally to this work.

\section{References}

1. Turkoglu M, Erdem GU, Suyani E, et al. Acute respiratory distress syndrome in patients with hematological malignancies. Hematology 18: 123-130, 2013.

2. Azoulay E, Mokart D, Lambert J, et al. Diagnostic strategy for hematology and oncology patients with acute respiratory failure: randomized controlled trial. Am J Respir Crit Care Med 182: 1038-1046, 2010.

3. Schuster DP, Marion JM. Precedents for meaningful recovery during treatment in a medical intensive care unit. Outcome in patients with hematologic malignancy. Am J Med 75: 402-408, 1983.

4. Gayados LA, Freireich EJ, Mantel N. The quantitative relation between platelet count and hemorrhage in patients with acute leukemia. N England J Med 266: 905-909, 1962.

5. Maschmer G, Beinert T, Buchheidt D, et al. Diagnosis and antimicrobial therapy of lung infiltrates in febrile neutropenic patients: guidelines of the infectious diseases working party of German society of haematology and oncology. Eur J Cancer 45: 2462-2472, 2009.

6. Ognibene FP, Martin SE, Parker MM, et al. Adult patients with severe neutropenia. N Engl J Med 315: 547-551, 1986.

7. Mokart D, van Craenenbroeck T, Lambert J, et al. Prognosis of acute respiratory distress syndrome in neutropenic cancer patients. Eur Respir J 40: 169-176, 2012.

8. Carlon GC. Admitting cancer patients to the intensive care unit. Crit Care Clin 4: 183-191, 1988.

9. Bird GT, Farquhar-Smith P, Wigmore T, Potter M, Gruber PC. Outcomes and prognostic factors in patients with hematological malignancy admitted to a specialist cancer intensive care unit: a 5 yr study. Br J Anaesth 108: 452-459, 2012.

10. Depuydt PO, Benoit DD, Vandewoude KH, et al. Outcome in noninvasively and invasively ventilated hematologic patients with acute respiratory failure. Chest 126: 1299-1306, 2004.

11. Hilbert G, Gruson D, Vargas F, et al. Noninvasive ventilation in immunosuppressed patients with pulmonary infiltrates, fever, and acute respiratory failure. N Engl J Med 344: 481-487, 2001.

12. Depuydt PO, Benoit DD, Roosens CD, Offner FC, Noens LA, Decruyenaere JM. The impact of the initial ventilatory strategy on survival in hematological patients with acute hypoxemic respiratory failure. J Crit Care 25: 30-36, 2010.

13. Depuydt PO, Benoit DD, Vandewoude KH, et al. Outcome in noninvasively ventilated hematologic patients with acute respiratory failure. Chest 126: 1299-1306, 2004.

14. Azoulay E, Lemiale V, Mokart D, et al. Acute respiratory distress syndrome in patients with malignancies. Intensive Care Med 40: 1106-1114, 2014.

15. Belenguer-Muncharaz A, Albert-Rodrigo L, Ferrandiz-Selles A, Cebrian-Graulleara G. Ten-year evolution of mechanical ventilation in acute respiratory failure in the hematological patient admitted to the intensive care unit. Med Intensiva 37: 452-460, 2013.

16. Soares M, Salluh JI, Spector N, et al. Characteristics and outcome of cancer patients requiring mechanical ventilator support $>24 \mathrm{~h}$. 
Intern Med 55: 1537-1545, 2016 DOI: 10.2169/internalmedicine.55.5822

Crit Care Med 33: 520-526, 2005.

17. Kress JP, Christenson J, Pohlman AS, Linkin DR, Hall J. Out- comes of critically ill cancer patients in a university hospital setting. Am J Respir Crit Care Med 160: 1957-1961, 1999.

(C) 2016 The Japanese Society of Internal Medicine http://www.naika.or.jp/imonline/index.html 